Fatigue and Alertness in the United States Railroad Industry Part II: Fatigue Research in the Office of Research and Development at the Federal Railroad Administration

\author{
Michael Coplen \\ U.S. Department of Transportation, \\ Federal Railroad Administration, \\ Office of Research and Development \\ Donald Sussman \\ U.S. Department of Transportation, \\ Research and Special Program Administration, \\ Volpe National Transportation Systems Center
}

\begin{abstract}
The Federal Hours of Service Act of 1907, which regulates the US railroad industry, imposes both maximum work hours and minimum rest periods. However, this act does not limit employees' weekly or monthly work hours, restrict the irregularity or unpredictability of on-call work schedules, or restrict mandatory commuting distances without compensatory time off. Extensive night work, irregular work schedules, extended work periods with few or no days off, and the policies, procedures, and agreements that encompass these work scheduling practices, all evolved within the limited provisions of this act. It is not clear, though, that broad changes in the hours of service laws are the answer to these problems. Consequently, the Office of Research and Development at the Federal Railroad Administration, with its Fatigue Research Program, has embarked upon a non-prescriptive approach to better manage fatigue in the railroad industry. This program includes the development and implementation of improved fatigue data collection methodologies, better measurement and evaluation tools, and more effective fatigue countermeasure strategies. The North American Rail Alertness Partnership (NARAP) has become an important means for understanding the fatigue-related problems in various operational settings, and for identifying specific programmatic areas that will better meet the needs of the industry. The program goals of improving the feasibility, utility and cost effectiveness of fatigue management are to be realized with the cooperative efforts of the government, unions, and the railroad industry, particularly though NARAP, and by broadly disseminating important technical findings through journal publications and conference proceedings.
\end{abstract}

Keywords: railroad, fatigue, alertness, shift work, work schedules. 


\section{Return to Top}

\section{BACKGROUND}

The Conference theme of non-prescriptive approaches to managing fatigue in transportation would normally present a unique challenge to government researchers, who work within a regulatory framework that requires scientific and technical support for its rulemaking activities. Fortunately, a new approach for improving safety in the U.S. railroad industry was initiated in the 1990's by the Federal Railroad Administration (FRA) under the administration of Joline Molitoris. As stated in a recent report by the General Accounting Office (1997), "Rather than citing violations and civil penalties as the primary means to obtain compliance with railroad safety regulations, FRA has emphasized cooperative partnerships with other federal agencies, railroad management, labor unions, and the states."

One of the outcomes of this new partnering approach to safety is the North American Rail Alertness Partnership (NARAP). As outlined in its charter, the purpose of NARAP is "to support industry-wide initiatives through the coordination, facilitation, and communication of efforts to reduce fatigue and promote safety in rail operations" and "to promote the safety of the industry's employees by developing effective countermeasures, based upon analytical and/or scientific data" (1998). Formally organized in 1998, NARAP members include key government, labor and management officials, who meet on a quarterly basis.

FRA's Office of Research and Development (OR\&D) has embarked upon a Fatigue Research Program. This program will systematically assess the many underlying factors that result in fatigue and reduced alertness, and develop appropriate tools that will assist the industry in developing its own effective fatigue management solutions. The program goals of enhanced alertness and performance are to be realized through FRA's cooperative approach with labor, management, and other government agencies, in particular NARAP. The Office of Research and Development OR\&D also expects to accomplish its program goals by broadly disseminating important technical findings. Three areas of research are needed to better understand fatigue problems and to help foster effective fatigue management programs in the U.S. railroad industry: (1) fatigue data collection, (2) valid and reliable fatigue measurement and evaluation tools, and (3) fatigue countermeasure strategies.

\section{$\underline{\text { Return to Top }}$}


As in most industries, consistent and reliable data on fatigue (particularly good exposure data) is often lacking in the railroad industry. For example, a recent study, entitled Switching Operations Fatalities Analysis: Findings and Recommendations from the SOFA Working Group (In Press), indicated that fatigue could not be investigated as a contributing factor to fatalities because the relevant data was missing, incomplete, or lacked good exposure measures for establishing appropriate rate information. Even though this study involved nearly two years of cooperative effort from management, labor, government and other human factors researchers, no definitive conclusions on fatigue-related fatalities could be established. Another preliminary study conducted by Foster-Miller, Inc. found FRA's accident/incident database useful for determining overall trends, but is limited in its usefulness for determining principal factors involved in incidents and injuries. Despite this, fatiguerelated incidents are generally considered to be much more prevalent than the data suggests (Sussman \& Coplen, 2000).

Incident Data and Incident Investigation Methods

Because there is limited fatigue-related data available for conducting good quality statistical analyses, the FRA is planning to modify its incident reporting system to include fatigue-related factors. The FRA is also planning to modify its incident investigation methods and training to recognize fatigue as a possible contributing factor to incidents. OR\&D plans to provide the required technical support needed for developing standardized fatigue data to be collected during incident investigations. OR\&D also plans to provide the necessary technical support for the development, design and utility testing of software tools needed for reliable and consistent collection of fatigue-related data.

Near-Miss Data

Efforts are also underway to evaluate the feasibility of developing a near-miss incident reporting system in the railroad industry. For every actual incident, many more near-miss incidents occur. With a much larger data set assumptions can be minimized, additional analyses can be conducted, and results can be interpreted with a greater degree of confidence. Although the FRA already requires the collection of near-miss data related to the safety conduct of locomotive engineers (Title 49 Code of Federal Regulations. Part 240 Section 309); fatigue-related analyses of this data set have not been conducted. Such data could be assessed by using tools such as those mentioned in the 'Work Schedule and Evaluation' section. This type of analysis could potentially indicate if there is any relationship between fatigue (determined from the pattern of work from the prior seven days) and near-miss occurrence. OR\&D is planning to evaluate the validity of available software programs that analyze fatigue as a possible contributing factor to both near-miss data and incident data. 
Work Schedule Data

OR\&D has begun a project to develop a database of railroad work schedules so that ergonomic assessments and comparisons of different work schedules on fatigue and performance can be conducted. This project will also provide important categorical information that can be included in any modifications to the incident reporting system.

Other Fatigue Data

Event recorders are the "black boxes" on locomotives that record most performance activity, including speed, throttle position, dynamic braking, air braking, etc. It is possible that these devices may be a useful source of data for evaluating the effects of different pilot projects on fatigue. To date, however, no known studies have utilized this method of data collection for evaluating the effectiveness of the field interventions currently being implemented by the industry. OR\&D will review the capability and practicality of this methodology for collecting fatigue-related data and evaluating the effectiveness of field intervention strategies.

OR\&D collects other fatigue data using a variety of research methods to build a broad base of knowledge on fatigue and its relationship to operational performance. Some of these methodologies include structured interviews, surveys, focus groups, work/rest activity diaries, wrist actigraphs, meta-analysis, naturalistic observations, Cognitive Task Analyses (CTA), and literature reviews.

OR\&D has compiled a comprehensive bibliography of scientific research on fatigue in railroad operations which is available on the FRA web site. The web site can be found at . This bibliography references only peer-reviewed articles in academic journals or academic books with railroad operating employees as subjects. A literature review is being conducted on the impact of extensive travel and commuting times on fatigue and performance in non-operating personnel. A bibliography of this literature will also be available on the above web site.

\section{$\underline{\text { Return to Top }}$}

\section{FATIGUE MEASUREMENT AND EVALUATION}

A systems approach for measuring and evaluating fatigue and its effects on operational performance is also needed. Research is being conducted in four major areas to develop valid and reliable tools that can aid in the measurement of fatigue and its effects on operational performance: (1) cognitive workload, taskload and performance modeling, (2) job analysis and staffing analysis, (3) work schedule evaluation, and (4) locomotive simulator experiments. 
Cognitive Workload, Taskload and Performance Modeling

Good predictive models of cognitive workload/taskload are needed to aid in the measurement of fatigue and to evaluate its effects on alertness and performance. Predictive models will also help evaluate the effects of changes in system states and enable "what if" type questions to be asked, so that fatigue and other performancerelated problems can be anticipated before they arise. Using these models, basic principles can be developed for systematically evaluating the effects of changing technology on operator performance and fatigue. These models and procedures for using them would then be made available to the industry.

It is important to use formalized methods, such as cognitive task analyses, to help develop good cognitive workload/taskload models and other decision-making and performance models, which can then be further tested and validated. CTA evolved as a collection of methodologies to enhance human performance by better understanding the cognitive decision-making processes of operations in complex environments, and thereby support the development of computer-based information processing tools. A wide variety of CTA techniques are often employed, including specific knowledge elicitation techniques, function-based task analyses, cognitive work analyses, concept mapping, and conceptual graph analyses. One of the most valuable aspects of conducting a CTA is the systematic documentation it provides on the inherent nature of complex cognitive tasks in operational settings. Therefore, CTAs are an important mechanism for developing measurement and evaluation tools for fatigue and its effects on operational performance.

OR\&D completed a preliminary CTA on railroad dispatchers to determine how experienced dispatchers manage trains and control track use (Roth, E.M., Malsch, N., Multer, J., and Coplen, M., 1999). The objective was to gain insight into the cognitive demands placed on railroad dispatchers and the decision strategies they have developed in response to those demands. Results of the CTA will be used to develop cognitive workload and performance models of dispatchers. New technology, such as Positive Train Control and other computer-based decision aid systems, can then be evaluated in the dispatching environment for their impact on mediating dispatcher fatigue. CTAs are being conducted for locomotive engineers, maintenance-of-way personnel and other non-operating employees.

On-going studies are being conducted to develop better measures of railroad dispatcher workload, stress, and fatigue, which can also be used in performance and alertness models. The purpose of this project is twofold: (1) to identify specific job task and work environment factors that contribute to the three variables of workload, stress, and fatigue among railroad dispatchers, and (2) to develop and validate appropriate methodologies for measuring each of these three variables in the 
dispatching environment. Issues being explored include differences among shifts, traffic volume effects and time of day effects on the level of dispatcher workload, stress, and fatigue.

In addition, a methodology is currently being devised for quantifying dispatcher taskload and objectively evaluating its effects on stress and fatigue. Once validation studies are complete, a PC-based system will be designed to assist in the uniform collection and analysis of taskload data. Such a PC-based system will benefit the industry by providing a ready-to-use electronic mechanism for tracking, monitoring, and evaluating dispatcher taskload. This project has been introduced to the industry at a kick-off meeting with labor and management representatives.

Job Analysis and Staffing Analysis

Job analysis research is being conducted to identify work practices and work environment conditions that contribute to employee fatigue, injuries and incidents so that remedial actions can be suggested. A preliminary study conducted by FosterMiller, Inc. established appropriate measures for evaluating incidents and injuries for employees on different work schedules and working conditions in yard and terminal operations. Fatigue-related findings suggest that certain yard job and work schedule categories experience significantly higher injury rates than the industry average for all railroad and all yard workers.

An integral component to work schedule evaluation and design is staffing analysis. Various staffing analysis software programs are being evaluated for their possible application to work schedule evaluation and design in the U.S. railroad industry. The goal is to develop a standard methodology for performing an objective staffing analysis in critical operations (locomotive engineers, dispatchers, etc.). This staffing analysis methodology will objectively determine minimum staffing levels needed as based on existing criteria. A kick-off meeting was recently held with labor and management to begin this process with dispatchers.

\section{Work Schedule Evaluation}

OR\&D continues to analyze fatigue and alertness data collected with work/rest activity diaries of on-call locomotive engineers (Pollard, 1996). Pilcher and Coplen (2000) evaluated how often and under what conditions locomotive engineers engage in work/rest cycles less than 24-hours, and what the effects of those work/rest cycles had on sleep quantity, sleep quality, and self-rated alertness. Further studies are being considered to evaluate the effect of these irregular work/rest cycles on fatigue and performance, including a review of relevant literature. 
Various alertness models and fatigue modeling software are also being evaluated for their application to the U.S. railroad industry. Similar to the fatigue modeling software developed in Australia for predicting work-related fatigue associated with specific rosters in the Australian railways (Dawson, Roach, Reid, and Baker, 1997; Fletcher and Dawson, 1998), OR\&D is developing a conceptual model for a software tool to evaluate work schedule data in the U.S. railroad industry. The goals are to develop an easy-to-use tool that will help railroad managers and workers in the U.S. railroad industry evaluate their own work schedules for their impact on alertness, and to aid in the design of more ergonomic work schedules. This PC software tool would be able to read work and sleep data from a variety of possible sources. It and produce predictions about alertness levels over the following 24-hours, as well as providing a method for easily producing raster-plot representations and deconstructing work schedule data. A research protocol will be designed to further develop and validate this tool using criteria based on one or more of available alertness and humanperformance models.

\section{Locomotive Simulator Experiments}

Simulator experiments allow researchers to explore important safety issues that may be impossible in field settings. Simulator research also provides a much higher degree of experimental control. Thus, they are an important component of OR\&D's Fatigue Research Program.

In a recent simulator study, Thomas Raslear and Kuehn (1997), found measurable decrements in train handling performance in work/rest cycles of less than 24-hours. In addition, they also found that these performance decrements were worse when the work/rest cycle was about 20 hours in length as opposed to 22 hours in length.

Napping is a potential technique for alleviating the impact of sleep deficits resulting from work/rest cycles of less than 24-hours. The FRA, with input from a number of international researchers, has designed a study to evaluate the effects of different nap durations on operation performance in an advanced high fidelity locomotive simulator.

Future simulator research is being planned to study the effects of one-person locomotive operations on fatigue and workload, and to evaluate the ability of cognitive displays, digital communications, and other technology applications to mediate fatigue-related performance problems in the locomotive cab.

Aspects of the physical environment impact operator fatigue and alertness. OR\&D is in the process of conducting a meta-analysis to investigate the relationship between temperature in the locomotive cab and operator performance. If it is concluded that 
such a relationship exists, follow-on simulator research will be considered for investigating the impact of this and other aspects of the physical working environment on crew fatigue and performance.

\section{$\underline{\text { Return to Top }}$}

\section{FATIGUE COUNTERMEASURE STRATEGIES}

NARAP identified eight key components for an effective fatigue countermeasure program in its Strategic Plan (1998), including: (1) education and training, (2) employee scheduling practices, (3) emergency response requirements, (4) alertness strategies, (5) evaluation of policies and procedures, (6) adequate rest environments, (7) work environment, and (8) implementation strategies. OR\&D's Fatigue Research Program provides technical support for many of NARAP's on-going strategies and activities. OR\&D regularly sponsors speakers at NARAP meetings to help educate its members about fatigue-related research methodologies and current findings. OR\&D is also actively involved in the NARAP Non-Operations Subcommittee, and will provide the technical support necessary to help answer the question, "Is there an increased risk of accidents/injuries associated with travel to and from work [in nonoperating personnel]?"

A number of other fatigue countermeasure strategies are also being planned or implemented by the FRA. OR\&D will be involved in evaluating the effectiveness of different fatigue countermeasure strategies and technologies employed by the industry.

Based on the results of its CTA studies and the development of appropriate models, OR\&D may evaluate, and possibly test: Positive Train Control, digital communication systems, advanced information displays, and other cognitive-based information systems for their ability to mediate the fatigue and alertness problems of locomotive engineers and dispatchers. OR\&D is also in the process of evaluating various alertness monitoring devices for their ability to predict fatigue problems, and their utility in allowing railroad operating personnel to take preventative measures to mitigate fatigue.

Finally, OR\&D is participating in a human factors program initiative by the U.S. Department of Transportation (DOT) to develop and implement tools, methods, and technologies to detect, measure, monitor, and mitigate operator fatigue in various transportation modes. The DOT released a Broad Agency Announcement soliciting proposals from individuals, businesses, academic institutions and other research centers. This solicitation on Operator Fatigue Management Analytic Systems and Technologies to Forecast and Manage Fatigue and Ensure Alertness for Commercial 
Transportation Operators was published in Commerce Business Daily on June 16, 2000 (Solicitation DTRS56-00-BAA-0007).

\section{$\underline{\text { Return to Top }}$}

\section{SUMMARY}

The Fatigue Research Program in FRA's Office of Research and Development encompasses a broad variety of data collection methodologies, measurement tools, and countermeasure strategies. Improving our methodologies and practices for collecting fatigue-related data will help us better understand the relationship between fatigue and specific work-related factors, such as work schedules, work environment, job task, or commuting. Improving our tools and methods for measuring and evaluating the effects of fatigue on operational performance will help the government and industry better estimate the true costs of fatigue. Locomotive simulator experiments, for example, that track and monitor fuel consumption, emergency brakings, braking frequency, and other operational performance measures, play a critical role in developing and implementing effective fatigue countermeasure strategies. These studies will not only help identify some of the cost factors associated with fatiguing work schedules or work environment, but will also help estimate the cost savings associated with implementing specific fatigue-related countermeasures, such as strategic napping.

In addition, the ability to quickly profile a specific work group or a particular operation, and then reasonably assess its susceptibility to fatigue, will help the government and industry prioritize and devote our resources to those areas that need them the most. The ability to reasonably forecast decrements in alertness and performance for individuals or work groups will help us develop more effective fatigue countermeasure strategies. The overall success of this program hinges not only on the systematic development of better measurement tools and evaluation methodologies, but also on the cooperation and teamwork of industry, government and research professionals alike. NARAP has become an important venue for enlisting these cooperative efforts. With this comprehensive Fatigue Research Program, and the support of NARAP, FRA's Office of Research and Development is committed to playing a leading role in developing non-prescriptive approaches to managing fatigue in transportation.

$\underline{\text { Return to Top }}$

REFERENCES 
Coplen, M. (1999). Compliance with railroad operating rules and corporate culture influences: Results of a focus group and structured interviews. (Report No. DOT/FRA/ORD-99-09; DOT-VNTSC-FRA-97-7). Washington, D.C.: U.S. Department of Transportation, Federal Railroad Administration.

Dawson, D., Roach, G. Reid, K., and Baker, A. (1997). Australian railways shiftwork and workload study: Final report. Woodville, SA: University of South Australia, The Centre for Sleep Research.

General Accounting Office (1997). Rail transportation. Federal Railroad Administration's new approach to railroad safety. (Publication No. GAO/RCED-97142). Gaithersburg, MD: U.S. General Accounting Office.

North American Rail Alertness Partnership strategic plan (1997, April). (Available from Jay Sorah, Department of Transportation, Federal Railroad Administration, Office of Safety, 331 Marshall Creek Road, Roanoke, Texas 76262.)

North American Rail Alertness Partnership charter (1998, April). (Available from Jay Sorah, Department of Transportation, Federal Railroad Administration, Office of Safety, 331 Marshall Creek Road, Roanoke, Texas 76262.)

Pilcher, J.J. and Coplen, M.K. (2000). Work/rest cycles in railroad operations: Effects of shorter-than 24-hour shift work schedules and on-call schedules on sleep. Ergonomics, 43, 573-588.

Pollard, J.K. (1996). Locomotive engineer's activity diary. (Report No. DOT/FRA/RRP-96/02; DOT-VNTSC-FRA-96-12). Washington, D.C.: U.S. Department of Transportation, Federal Railroad Administration.

Roth, E.M., Malsch, N., Multer, J., and Coplen, M. (1999, October). Understanding how railroad dispatchers manage and control trains: A cognitive task analysis of a distributed team planning task. Paper presented at the Annual Meeting of the Human Factors and Ergonomics Society. Houston, Texas.

Sherry, P. (2000, February). Current status of fatigue countermeasures in the railroad industry. Speech at the North American Railroad Alertness Partnership. Washington, D.C. Denver, CO: University of Denver, Intermodal Transportation Institute, Counseling Psychology Program.

Switching operations fatalities analysis ( SOFA): Findings and recommendations from the SOFA Working Group (In Press). Washington DC: FRA 
Sussman, D. and Coplen, M. (2000, March). Fatigue and alertness in the United States railroad industry. Part I: The nature of the problem. Paper presented at the $4^{\text {th }}$ International Conference on Fatigue and Transportation: Coping with the 24-hour society. Fatigue management alternatives to prescriptive hours of service, Fremantle, Western Australia.

Thomas, G., Raslear, T., and Kuehn, G. (1997). The effects of work schedule on train handling performance and sleep of locomotive engineers: A simulator study. (Report No. DOT/FRA/ORD-97-09). Washington, D.C.: U.S. Department of Transportation, Federal Railroad Administration. 